\title{
The influence of buoyant convection on the nucleation of n- propanol in thermal diffusion cloud chambers
}

\author{
Frank T. Ferguson \\ Richard H. Heist \\ Fairfield University, rheist@fairfield.edu \\ Joseph A. Nuth
}

Follow this and additional works at: https://digitalcommons.fairfield.edu/engineering-facultypubs Copyright 2010 American Institute of Physics

The final publisher PDF has been archived here with permission from the copyright holder. https://doi.org/10.1063/1.3429618

\section{Peer Reviewed}

\section{Repository Citation}

Ferguson, Frank T.; Heist, Richard H.; and Nuth, Joseph A., "The influence of buoyant convection on the nucleation of n-propanol in thermal diffusion cloud chambers" (2010). Engineering Faculty Publications. 146.

https://digitalcommons.fairfield.edu/engineering-facultypubs/146

\section{Published Citation}

Ferguson, F. T., Heist, R. H., \& Nuth, J. A. (2010). The influence of buoyant convection on the nucleation of npropanol in thermal diffusion cloud chambers. The Journal of chemical physics, 132(20), 204510. doi:10.1063/ 1.3429618.

This item has been accepted for inclusion in DigitalCommons@Fairfield by an authorized administrator of DigitalCommons@Fairfield. It is brought to you by DigitalCommons@Fairfield with permission from the rightsholder(s) and is protected by copyright and/or related rights. You are free to use this item in any way that is permitted by the copyright and related rights legislation that applies to your use. For other uses, you need to obtain permission from the rights-holder(s) directly, unless additional rights are indicated by a Creative Commons license in the record and/or on the work itself. For more information, please contact digitalcommons@fairfield.edu. 


\section{The influence of buoyant convection on the nucleation of $n$-propanol in thermal diffusion cloud chambers}

Frank T. Ferguson, Richard H. Heist, and Joseph A. Nuth

Citation: The Journal of Chemical Physics 132, 204510 (2010); doi: 10.1063/1.3429618

View online: https://doi.org/10.1063/1.3429618

View Table of Contents: http://aip.scitation.org/toc/jcp/132/20

Published by the American Institute of Physics

\section{Articles you may be interested in}

Stable stratification alteration in a thermal diffusion cloud chamber

The Journal of Chemical Physics 113, 8085 (2000); 10.1063/1.1315358

A Reference Equation of State for the Thermodynamic Properties of Sulfur Hexafluoride $\left(S F_{6}\right)$ for Temperatures from the Melting Line to $625 \mathrm{~K}$ and Pressures up to $150 \mathrm{MPa}$ Journal of Physical and Chemical Reference Data 38, 33 (2009); 10.1063/1.3037344

The Nucleation of Ice Formation by Silver lodide

Journal of Applied Physics 18, 593 (1947); 10.1063/1.1697813

The flow diffusion nucleation chamber: A quantitative tool for nucleation research

The Journal of Chemical Physics 104, 382 (1996); 10.1063/1.470837

Experimental studies of the vapor phase nucleation of refractory compounds. IV. The condensation of magnesium

The Journal of Chemical Physics 104, 3205 (1996); 10.1063/1.471085

Review of Vapor to Liquid Homogeneous Nucleation Experiments from 1968 to 1992

Journal of Physical and Chemical Reference Data 23, 781 (1994); 10.1063/1.555951

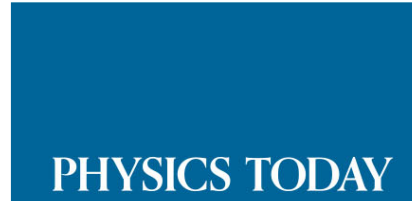

ADVANCED LIGHT CURE ADHESIVES 


\title{
The influence of buoyant convection on the nucleation of $n$-propanol in thermal diffusion cloud chambers
}

\author{
Frank T. Ferguson, ${ }^{1, a)}$ Richard H. Heist, ${ }^{2}$ and Joseph A. Nuth ${ }^{3}$ \\ ${ }^{1}$ Department of Chemistry, Catholic University of America, 620 Michigan Avenue, Washington 20064, USA \\ ${ }^{2}$ College of Engineering, Embry-Riddle Aeronautical University, Daytona Beach, \\ Florida 32114-3900, USA \\ ${ }^{3}$ Code 691, NASA-Goddard Space Flight Center, Greenbelt, Maryland 20771, USA
}

(Received 30 September 2009; accepted 22 April 2010; published online 28 May 2010)

\begin{abstract}
A two-dimensional numerical model has been applied to three thermal diffusion cloud chamber (TDCC) investigations of $n$-propanol in helium taken by two different research groups to provide a quantitative example of how the results in these chambers can be affected by buoyant convection. In the first set of TDCC data, corrections for buoyancy resolve an apparent discontinuity in critical supersaturation data and also yield nucleation rate data that tend to agree better with higher rate, expansion-based studies at the same temperature. In the second TDCC study, the nucleation of propanol was studied over an extended pressure range. When the model was applied to these data, the possible variation in supersaturation values due to convection induced by conditions at the chamber sidewall was found to be comparable in magnitude to the experimentally observed range and may be responsible for some of this observed pressure dependence. In the third TDCC study, the combination of an error in a transport property and buoyant convection appear responsible for a perceived pressure effect in the experimental data. After correcting for this transport property and for buoyancy, the results at higher temperatures agree quite closely with the predictions of classical nucleation theory. (C) 2010 American Institute of Physics. [doi:10.1063/1.3429618]
\end{abstract}

\section{INTRODUCTION}

For some time there has been an effort to understand the carrier gas or pressure effect in vapor phase nucleation studies. This effect refers to a change in nucleation rate with pressure or type of background gas even though such an effect is not predicted by classical nucleation theory (CNT). Previously, it was assumed that the only role the carrier gas played in the nucleation process was to establish a temperature field for the growing clusters. Depending on experimental conditions, this pressure effect may be positive (nucleation rate increasing with the amount of background gas), negative, or the dependence may be negligible. The pressure effect has been observed in diffusion-based experimental devices such as the thermal diffusion cloud chamber (TDCC) and the laminar flow diffusion cloud chamber, (LFDC), as well as in some expansion-based devices.

There have been relatively few studies of nucleation over extended ranges of pressure. Heist et al. studied the nucleation of several alcohols up to $4 \mathrm{MPa}$ in a high pressure version of the TDCC. ${ }^{1,2}$ Measured supersaturations were very pressure dependent, increasing with increasing pressure, and this pressure dependence was also more pronounced at lower nucleation temperatures. In addition, this pressure dependence was influenced by the type of carrier gas. Heist et $a l$. found the pressure dependence to be stronger when helium was used as the background gas rather than hydrogen.

Luijten $e t a l .^{3}$ studied the nucleation of water in helium and nitrogen at $1,2.5$, and $4 \mathrm{MPa}$ and $n$-nonane in helium

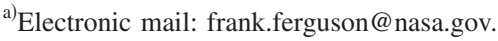

and methane at these same pressures in a pulse expansion wave tube. Only weak pressure effects were observed with helium as the background gas, but strong pressure effects were observed for both nitrogen and methane. Peeters et $a l^{4}$ later improved the measurements of $n$-nonane in methane and also performed pressure studies of water nucleation in methane. The observed pressure dependence compared very favorably with a model developed by Luijten et al. ${ }^{5}$ that incorporated the influence of the carrier gas into nucleation theory. In this theory, the pressure effect arises from two sources: the first due to an increase in the saturated vapor density with pressure and the second due to a decrease in the droplet surface tension with pressure. These pressure effects are very dependent on the type of carrier gas and are observable over very extended ranges of pressures (e.g., $\sim 1 \mathrm{MPa}$ ), much higher than the approximately atmospheric pressure range of the TDCC where an observable pressure effect is still seen.

Several other explanations have been proposed to explain this carrier gas effect including nonideal gas effects, ${ }^{6}$ aspects not included in nucleation theories, ${ }^{7,8}$ errors in the droplet or particle flux detection, ${ }^{9}$ experimental artifacts due to incomplete modeling of the chamber, ${ }^{10-13}$ and diffusional contributions to the growing droplet. ${ }^{14}$ Brus et al. ${ }^{15}$ compiled a very complete history of the pressure effect and the reader is urged to reference this work for more detail. Only some highlights from the most recent works regarding the pressure effect will be described here.

There have been a number of molecular dynamics simulations recently related to the role of the carrier gas as a thermalyzing medium as well as studies of the evaporation 
rates of clusters. Merikanto et al. ${ }^{16}$ performed a Monte Carlo study of argon nucleation rates under both equilibrium and nonequilibrium conditions. Tang and Ford ${ }^{17}$ studied the evolution of single argon clusters using molecular dynamics over long periods and found a stabilizing effect on the clusters that varied nonlinearly with the amount of background gas. Yosouka and Zeng ${ }^{18}$ simulated vapor phase nucleation using three different carrier gas models and found the results to be strongly dependent on the choice of model, noting either a positive or negligible pressure effect. Wedekind et al. ${ }^{19}$ studied the role of the carrier gas as a thermostat via molecular dynamics. Although some deviations were noted, these variations amounted to approximately a factor of 2 difference in nucleation rate and were essentially negligible. Barrett ${ }^{20}$ performed a stochastic simulation of nonisothermal nucleation and his results gave a variation in nucleation rate with supersaturation that depends on the thermalyzing gas.

Very recently, Wedekind et al. ${ }^{21}$ revisited the equations used in developing CNT and incorporated two competing contributions arising from the background gas: one related to nonisothermal effects and the efficiency of thermalization and another that arises from the additional work needed to grow the cluster in the presence of the carrier gas. Interestingly, based on different conditions, the model predicts the possibility of a positive effect, a negative effect, and at times no effect at all, similar to what is reported in experimental investigations.

The carrier gas effect has been studied extensively recently with the LFDC combined with great improvements in the modeling and understanding of these devices. Mitrakos et al. $^{22}$ developed a more comprehensive model of the LFDC that included aerosol dynamics and compared the results with the more typical model that ignores these processes. Their comparison showed essentially negligible differences between the two, thereby justifying the use of the simpler model for LFDC calculations. Hyvärinen et al. ${ }^{23,24}$ initially reported a pressure effect with $n$-butanol in helium in a LFDC, but after comparison of the chamber model with a commercial based code, they found that this effect was attributable to an error in a transport property. Herrmann et al. ${ }^{25,26}$ compared the model of the LFDC developed by their group with the results obtained from a commercial, computational fluid dynamics code and found that both models agreed within the uncertainty limits of the experimental device. Most recently, a pressure effect for $n$-pentanol in helium and $n$-pentanol in argon has been observed in LFDCs ${ }^{26,27}$ For helium, a positive pressure effect was observed at all of the experimental temperatures while both positive and negative effects were seen for $n$-pentanol in argon.

In 2006, Brus et al. ${ }^{15}$ presented a study of $n$-propanol nucleation in a TDCC. These authors reported a pressure effect and found the nucleation rate increasing with decreasing chamber pressure. Buoyant convection in the TDCC induced by conditions at the chamber sidewall has been theorized to affect the supersaturation and temperature profiles calculated via the typical one dimensional (1D) model and may be related to the pressure effect. Brus et al. compared their 1D calculations with a two-dimensional (2D) model to verify that the pressure effect was real and not an experimental artifact.

Buoyant convection and its role in the interpretation of TDCC results has been studied by several groups in the past, ${ }^{11-13,28}$ and it may play a significant role in the observed pressure effect in TDCCs. The observed carrier gas effect has several characteristics that are also similar to what one would expect due to buoyancy. For example, buoyancy would tend to increase with lower temperatures and will depend on both the type and amount of background gas, similar to that typically observed for the pressure effect. Flows predicted to occur within the chamber are often very subtle, making them difficult to detect, yet they can alter the temperature and supersaturation profiles in the center of the chamber. These flows are induced by conditions at the chamber sidewall and the nucleation rate within the chamber has been noted to depend on the applied heating at the sidewall. ${ }^{29}$ Many theories developed to explain the pressure effect are successful in predicting an effect, yet the expected magnitude is often much lower than observed. In contrast, the magnitude of the pressure effect is well within the range possible due to buoyant convection. For example, Ferguson et al. ${ }^{11}$ noted that for larger TDCCs, it is possible to change the nucleation rate within the center of the chamber by over ten orders of magnitude based on whether the sidewall is heated or not.

Previous works on buoyant convection have typically provided insight on how these flows occur and guidelines for minimizing them. The goal of this work is to apply a model of these flows to experimental data, recalculating the results to account for buoyant flows and giving quantitative examples of what effect these flows can have. In this particular case the model will be applied to three TDCC investigations of $n$-propanol in helium. One goal of experimental nucleation studies is to provide data that can be used to test theories over wide ranges. As noted in a recent review by Anisimov et al., ${ }^{30}$ one of the challenges of vapor-gas/liquid nucleation experiments is that substantial inconsistencies have been reported among experimental data gathered using different experimental devices. For propanol, there is some overlap between the experimental temperature ranges of the TDCC and other expansion-based studies. Therefore, an additional goal of this work is to compare the newly recalculated, TDCC nucleation data with expansion studies to see if buoyant convection could be responsible for some of these inconsistencies.

\section{MODEL OVERVIEW}

The 2D model of the TDCC used here is similar to the model reported earlier. ${ }^{11}$ In principle, this model is very much like that used in the typical 1D modeling of the chamber with added dimensionality and the addition of the momentum equation. In the typical 1D modeling of the chamber, the vapor species balance equation is written in terms of mole fractions. Since mass average velocity components are needed for the momentum equation, it is more convenient to write the vapor concentration equation in terms of mass fraction. 
The total mass flux of the vapor (component $A$ ) with respect to the mass average velocity of the system, $\mathbf{j}_{A}$, is given by two components,

$$
\mathbf{j}_{A}=\mathbf{j}_{A}^{(x)}+\mathbf{j}_{A}^{(T)} \text {. }
$$

The term $\mathbf{j}_{A}^{(x)}$ is simply the ordinary diffusive flux while the second term $\mathbf{j}_{A}^{(T)}$ is the mass flux induced by the thermal gradient, i.e., the Soret effect. In terms of the mass fractions, these fluxes are given by the two terms of Eq. (2), respectively,

$$
\mathbf{j}_{A}=\left[-\rho D_{A B} \nabla w_{A}\right]+\left[-\rho D_{A B} \alpha_{T} w_{A}\left(1-w_{A}\right) \nabla \ln T\right],
$$

where $w_{A}$ is the mass fraction of the vapor, $T$ the temperature, $\rho$ the density of the carrier gas/vapor mixture, $\alpha_{T}$ the thermal diffusion coefficient, and $D_{A B}$ the binary diffusion coefficient for the vapor-gas mixture.

The energy flux with respect to the mass average velocity of the system, $\mathbf{q}$, is composed of the three terms,

$$
\mathbf{q}=\mathbf{q}^{(\mathbf{c})}+\mathbf{q}^{(\mathbf{d})}+\mathbf{q}^{(\mathbf{x})} \text {. }
$$

The term $\mathbf{q}^{(\mathbf{c})}$ is the ordinary, conductive flux, $\mathbf{q}^{(\mathbf{d})}$ is the species interdiffusion flux, and $\mathbf{q}^{(\mathbf{x})}$ is the Dufour energy flux, the energy flux term arising from the concentration gradient. In terms of temperature and enthalpies of the system, these three individual fluxes are given by the terms in Eq. (4), respectively,

$$
\mathbf{q}=[-k \nabla T]+\left[\left(h_{A}-h_{B}\right) \mathbf{j}_{A}\right]+\left[\alpha_{T} \frac{R T M^{2}}{M_{A} M_{B}} \mathbf{j}_{A}\right],
$$

where $k$ is the mixture thermal conductivity, $h_{A}$ and $h_{B}$ the specific enthalpies of the vapor and background gas, respectively, $R$ the ideal gas constant, and $M_{A}, M_{B}$, and $M$ the molecular weight of the vapor, the background gas, and mixture, respectively.

The energy and mass fluxes above are for a reference frame moving with the mass average velocity of the system, u. To change the reference frame to a fixed reference frame, the convective fluxes are added to each equation to give the following.

Vapor mass fraction.

$$
\nabla \cdot\left(\rho \mathbf{u} w_{A}\right)=-\nabla \cdot \mathbf{j}_{A} .
$$

Energy equation.

$$
\nabla \cdot(\rho \mathbf{u} h)=-\nabla \cdot \mathbf{q},
$$

where $h$ is the specific enthalpy of the vapor/carrier gas mixture. To complete the model, the continuity and momentum equations are needed.

\section{Continuity equation.}

$\nabla \cdot(\rho \mathbf{u})=0$.

\section{Momentum equation.}

$$
\nabla \cdot(\rho \mathbf{u u})=-\nabla \cdot \tau-\nabla P+\rho \mathbf{g},
$$

where $\tau$ is the stress tensor, $P$ the pressure, and $\mathbf{g}$ the gravitational acceleration. The stress tensor components relating the stress tensor to the individual velocity components can be found in Ref. 28 or 31. Equations (5)-(8), combined with the ideal gas equation of state, are used to compute the profiles within the TDCC. If only a 1D solution were needed, Eqs. (5)-(7) would suffice and are completely equivalent to the typical 1D model used to analyze TDCC data. In a 2D model, the momentum equation is needed to account for the radial component of velocity as well as the axial component.

Boundary conditions for the chamber sidewall are the driver for buoyant convection within the TDCC and will be discussed in detail in a later section. Boundary conditions for the top and bottom plates for the current model are identical to the 1D model, e.g., that the vapor is saturated at the temperature of these two plates. In the typical, mole-fraction based 1D model of the TDCC, since there is no net transport of the background gas at the top and bottom liquid surfaces, by continuity the background gas remains stagnant and this assumption is used to simplify the vapor transport equation. Likewise, in the 2D modeling, it is assumed that the flux of the background gas is zero. Therefore,

$$
n_{B \eta}=j_{B \eta}+\rho u_{\eta} w_{B}=0,
$$

where $n_{B \eta}$ is the total (convective + diffusive) mass flux of the background component, $B, w_{B}$ the mass fraction of the background gas, and $\eta$ is used to denote the direction normal to the liquid surface. The total diffusive flux of the background gas in the direction $\eta$ denoted by $j_{B \eta}$ is a combination of the Soret and ordinary diffusive fluxes. It should be noted that while there is still no net transport of carrier gas at the boundaries of the 2D model, the carrier gas is not necessarily stagnant in this case. With the added dimensionality, the carrier gas is able to recirculate. ${ }^{32}$

This equation for the flux of the background gas can be rearranged to give for the binary mixture,

$$
u_{\eta}=-\frac{j_{B \eta}}{\rho w_{B}}=\frac{j_{A \eta}}{\rho\left(1-w_{A}\right)},
$$

where $j_{A \eta}$ is calculated via Eq. (2). Equation (10) is used as the boundary condition for the velocity components normal to all liquid surfaces. For the velocity components tangential to the chamber surfaces where there is often a very thin layer of liquid, the velocity should be calculated by matching the tangential stress at the vapor liquid interface. Because this liquid film is typically thin, it is assumed that this boundary condition can be replaced with the no-slip boundary condition. At the chamber centerline, the radial gradients of mass, energy, and the axial velocity component are zero while the radial velocity component is zero.

Again, at the chamber sidewall, it assumed that the vapor is saturated at the temperature of the sidewall. The temperature profile, whether heated or not, at the chamber sidewall is the driving force behind convection within the TDCC so this will be discussed in greater detail in Sec. III. Equations (5)-(8) are solved using finite differences on a rectangular grid using the control volume technique, SIMPLER, developed by Patankar. ${ }^{33}$ These equations are iteratively solved until the mass conservation equation for each control volume is reached within a suitably small criteria. 


\section{SIDEWALL BOUNDARY CONDITIONS}

As mentioned earlier, the boundary conditions at the chamber sidewall are very important since these conditions are the drivers for buoyant convection in the chamber. In the past, TDCCs have been operated with two kinds of conditions at the sidewall termed "wet" or "dry." Dry operation is the most common, and in this case the chamber sidewalls are heated to prevent condensation of the working fluid on the inner walls of the chamber. This operation provides the least disturbance to the concentration field since there is no flux of condensable to/from the chamber wall. A temperature gradient, and hence a density gradient, does exist at the wall. To minimize the possibility of convection within the chamber, the chamber is heated as little as possible while still keeping the condensing vapor slightly undersaturated at the wall and free of liquid. Often, this ideal heating of the chamber wall is done by using different heating zones to minimize the possibility of overheating the chamber wall. In the model, this dry operation is approximated by first specifying that there is no mass flux at the chamber sidewall. Based on this concentration profile, the temperature along the wall is then adjusted so that every point is just saturated, i.e., that the supersaturation is exactly one at every point.

In wet operation, the chamber walls are not heated and the condensable vapor is simply allowed to condense on the sidewalls. While this operation provides a much smaller temperature gradient at the sidewall, there is now a condensable vapor concentration gradient at the wall. In the model, this wet wall operation is approximated by simply assuming a linear temperature drop along the wall. This linear temperature drop is based on the assumption that conduction along the sidewall between the top and bottom plates is the dominant heat transfer mechanism. For the vapor mass fraction boundary condition, it is assumed that the vapor is again saturated and calculated from the equilibrium vapor pressure at each computed temperature along the wall.

Both operating modes will produce a density gradient at the wall. In cases where such density differences occur, buoyant or natural convection will work to stratify these gradients. Therefore, in both operating modes there can exist a rising flow very near the chamber sidewall. This upward flow is then balanced by a broader, but weaker downward flow in the chamber interior.

The question now becomes which mode is best? Again, as noted earlier, dry operation is the most common. Fisk et al. ${ }^{29}$ have demonstrated that in the vicinity of ideal heating there exists a plateau in the nucleation rate- a region where changes in nucleation rate within the TDCC are relatively insensitive to modest changes in heating at the chamber sidewall. This condition changes sharply with little heating (corresponding to wet wall operation) or to overheating of the chamber wall, where the nucleation rate can be significantly different. Fisk et al. offered this as evidence that dry operation provided the least disturbance to the interior chamber conditions and this idea was also supported by later modeling of the chamber. ${ }^{11}$

As further evidence that dry operation is preferable to wet, consider the following. In numerical modeling of natu- ral convective flows, the Boussinesq approximation is often used. In this approximation, all physical properties are considered constant in the relevant equations except where the density appears in the body force term of the momentum equation, $\rho \mathbf{g}$. In this case, the density is replaced by a linear approximation. For example,

$$
\rho=\rho_{0}\left[1-\beta_{T}\left(T-T_{0}\right)-\beta_{w}\left(w_{A}-w_{A 0}\right)\right],
$$

where

$$
\beta_{T}=-\left.\frac{1}{\rho_{0}}\left(\frac{\partial \rho}{\partial T}\right)\right|_{0}
$$

and

$$
\beta_{w}=-\left.\frac{1}{\rho_{0}}\left(\frac{\partial \rho}{\partial w_{A}}\right)\right|_{0},
$$

where $\beta_{T}$ is defined as the thermal expansion coefficient and $\beta_{w}$ is the solutal expansion coefficient and the subscript 0 denotes a reference condition. One advantage of the Boussinesq approximation is that it greatly reduces the complexity involved in modeling of buoyant flows. Unfortunately, the Boussinesq approximation is only appropriate for systems where density changes are very minor and the product of the expansion coefficient and the driving force is much less than 1, e.g., $\beta_{T}\left(T-T_{0}\right) \ll 1 .^{34}$ In 1985 , Kacker and Heist made a set of critical superaturation measurements in a TDCC for $n$-propanol in helium. Using these data as an example, the value of $\beta_{T}\left(T-T_{0}\right)$ ranges from 0.15 to 0.17 while $\beta_{w}\left(w_{A}\right.$ $-w_{A 0}$ ) ranges from 0.20 to 0.99 . This example clearly demonstrates that the Boussinesq approximation would likely not be appropriate for quantitative calculation of the supersaturation profiles within the TDCC. Despite this shortcoming, this example does provide some information on the scope of density changes within the TDCC due to thermal and concentration differences. Since both wet and dry conditions produce similar density gradients at the chamber sidewall, this would suggest that the chamber would be less susceptible to convection from temperature disturbances (dry wall operation) than concentration-induced ones (wet wall operation). This very simple analysis also agrees with more complex modeling and experimental observations. ${ }^{11,29,35}$

\section{AXIAL FLOW IN THE TDCC}

Before continuing to the quantitative calculations of the TDCC data it is perhaps helpful to provide a detailed review of how buoyant convection can influence the temperature and concentration profiles within the chamber. Kacker and Heist ${ }^{36}$ provided a set of critical supersaturation data for $n$-propanol over the temperature range of $260-310 \mathrm{~K}$ as well as nucleation rate measurements for three isotherms in this temperature range. For this example a critical supersaturation experimental point at the midpoint of the temperature range is used as an example. This point corresponds to run 10 with a bottom plate temperature of $310.1 \mathrm{~K}$, an upper plate temperature of $264.6 \mathrm{~K}$, and a total pressure of $133.3 \mathrm{kPa}$ with helium as the carrier gas.

Convection within the center of the chamber depends on several factors. The advantage of having a model of this 

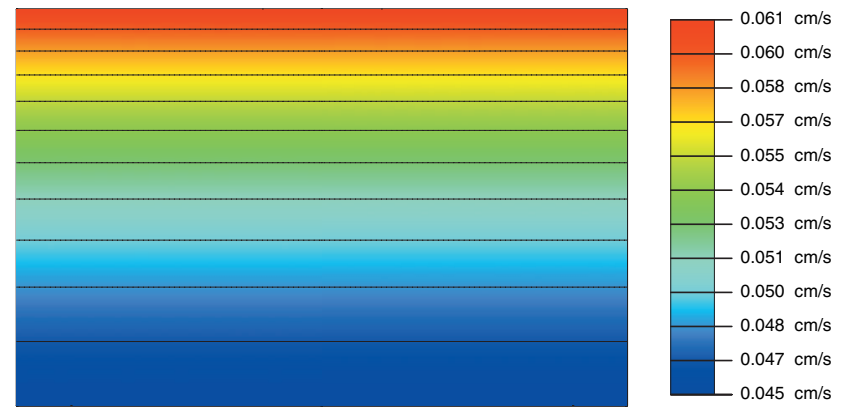

(a) 1D Solution
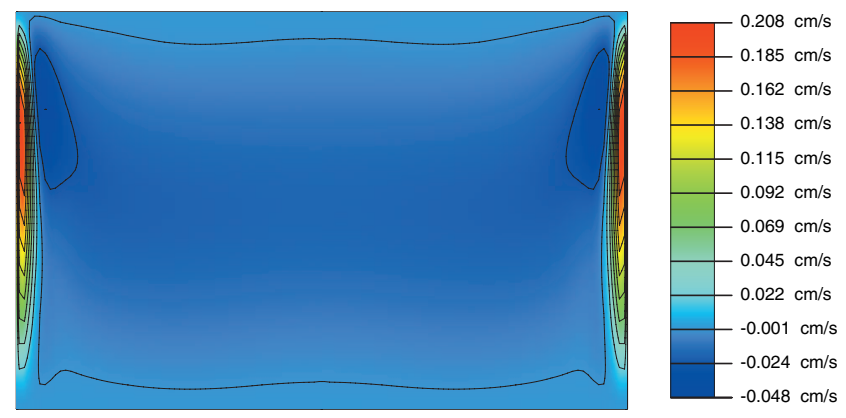

(c) 2D, Buoyancy with no Stefan flow

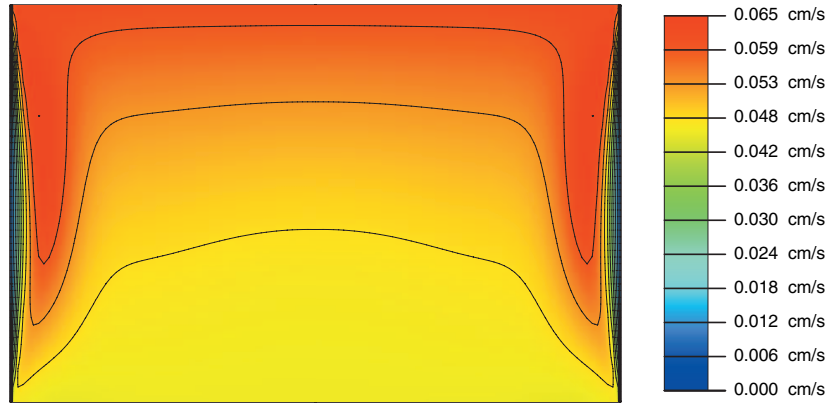

(b) 2D, Stefan flow with no buoyancy

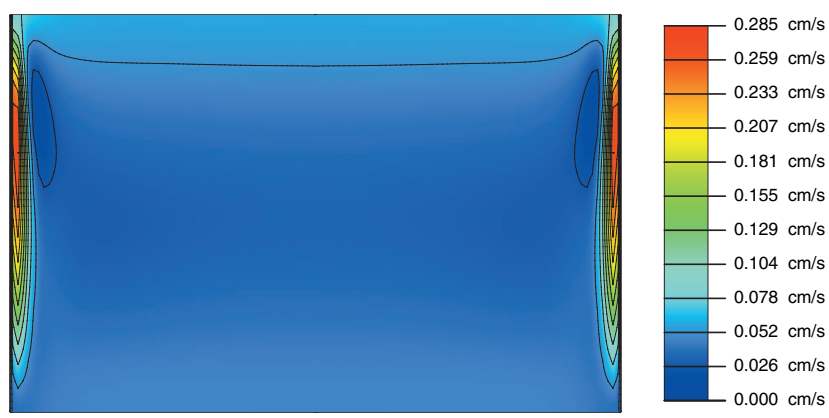

(d) Full 2D solution

FIG. 1. Contour plots of the axial velocity component within the TDCC under various conditions. (a) is the velocity profile calculated via the typical, 1D model of the chamber. (b) is the velocity profile within the chamber after adding the sidewall boundary, but does not include any buoyancy-induced flows. (c) is a 2D calculation of the velocity profile within the chamber based on buoyancy alone. (d) is a combination of the two convective flows shown in (b) and (c), giving the full $2 \mathrm{D}$ solution to the chamber velocity profile.

system is that this system can be decomposed into its constituent parts to see the relative effect of each piece. Buoyant convection typically acts to slightly reduce the convective energy and mass fluxes within the center of the TDCC. Therefore, the focus of this example will be on the axial velocity component within the chamber.

The first example is given in Fig. 1(a). This contour plot is a $2 \mathrm{D}$ representation of the axial velocity profile that would be predicted by the typical $1 \mathrm{D}$ model of the TDCC. This example can be made by simply removing the influence of the chamber sidewall, i.e., the concentration and temperature gradients have been set to zero at the wall. There exists a weak, upward flow at every point in the chamber, changing slightly in magnitude with the density differences within the chamber as dictated by the continuity equation. The conditions in this case are similar to those found in a Stefan tube where the background carrier gas is stagnant and there is a weak convective flow of the condensable vapor, labeled as the Stefan flow in this case. ${ }^{37}$

In Fig. 1(b), the influence of the chamber sidewall is included, but the influence of buoyancy is not. In the model, these buoyancy effects can easily be eliminated by setting the gravitational level to zero. In this case, the concentration gradient at the wall is zero and the sidewall is assumed to be ideally heated, i.e., that the temperature is set to the value where the vapor is just saturated at every point along the wall. Additionally, at the sidewall, the axial velocity component is set to zero by assuming the no-slip boundary condition at this surface. With the addition of this zero value at the wall, there is a gradient in the axial velocity at the sidewall and the contour levels are rescaled. Therefore, there is a reduction in the number of contours within the center of the chamber in Fig. 1(b) over Fig. 1(a). Nevertheless, the contours within the center of the chamber are still relatively flat and of similar magnitude as the previous 1D case.

In Fig. 1(c), the effect of buoyancy alone is examined. The sidewall boundary conditions are identical to those of Fig. 1(b), but the so-called Stefan flow has been eliminated by setting the axial velocity components at the top and bottom surfaces equal to zero instead of using Eq. (10). The temperature gradient at the sidewall simply drives a recirculation of the fluid within the chamber. A thin, but strong upward flow at the sidewall is balanced by a broad, weak downward flow in the inner part of the chamber. There are also negative velocity values within the chamber in this case.

Finally, both the aspects of cases (b) and (c) are combined in the full 2D solution of the chamber as given in Fig. 1(d). One point to note is that although buoyancy does generate a downward component to the flow as given in Fig. 1(c), it does not change the direction of the flow in the center of the chamber. At no point in the chamber is the axial component downward; there is always a net convective flux from the bottom plate to the top of the chamber.

Although these contour figures are useful in visualizing the location and extent of the flows, the levels in the region of interest, the chamber centerline, are masked by the extreme values at the chamber sidewall. Therefore, results from 


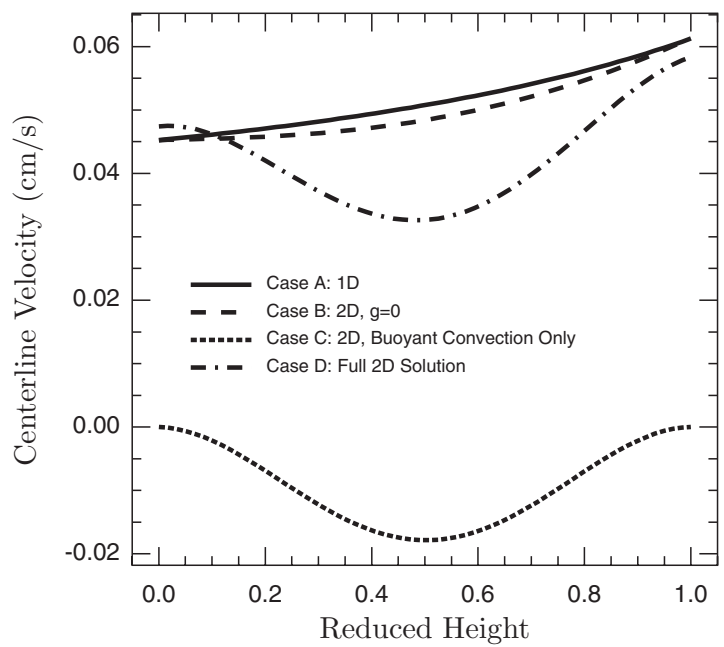

FIG. 2. TDCC centerline axial velocity as calculated from various models of the chamber.

each case are also presented in Fig. 2. In this figure, only the axial velocities along the centerline for each case are plotted.

In case (a), the typical 1D solution, there is a slowly varying increase in velocity from the bottom to the top plate, and, as noted before, this variation is due to the changing density profile. Interestingly, even in case (b) where buoyancy is neglected, the "addition" of the chamber sidewall slightly reduces this 1D convective flux. Stefan flow is not included in case (c), the buoyancy-only calculation. Therefore, the centerline flow in this case is always less than or equal to zero.

In the full solution, case (d), the axial velocities at the top and bottom boundaries are similar to the 1D solution, but there is a reduction in the flow in the center of the chamber. The velocities at the boundaries do not match the 1D solution because the buoyant flow also influences the concentration gradients at these boundaries, changing the boundary conditions given in Eq. (10).

Admittedly, the centerline velocity components are very weak. Yet these convective contributions play an important role in the accurate calculation of the supersaturation profile. Neglecting these convective contributions can lead to significantly lower calculated chamber supersaturations. ${ }^{32}$ Likewise, a reduction in these fluxes by buoyancy can lead to a reduction in the supersaturation profile within the chamber center. Brus et al. ${ }^{15}$ noted that buoyancy tends to distort the velocity vectors in the region of the sidewall, but that the ones in the chamber center remain vertical and relatively unaffected. This occurs because the small reductions in velocity at the centerline are masked by the larger values at the sidewall. While the velocity vectors at the centerline may at first glance seem to be unaffected, buoyancy does reduce their magnitude slightly over the values calculated via the typical 1D model.

\section{1985 PROPANOL TDCC MEASUREMENTS}

As noted earlier, Kacker and Heist made a set of critical supersaturation measurements for $n$-propanol over the temperature range of approximately $260-310 \mathrm{~K}$ as well as rate

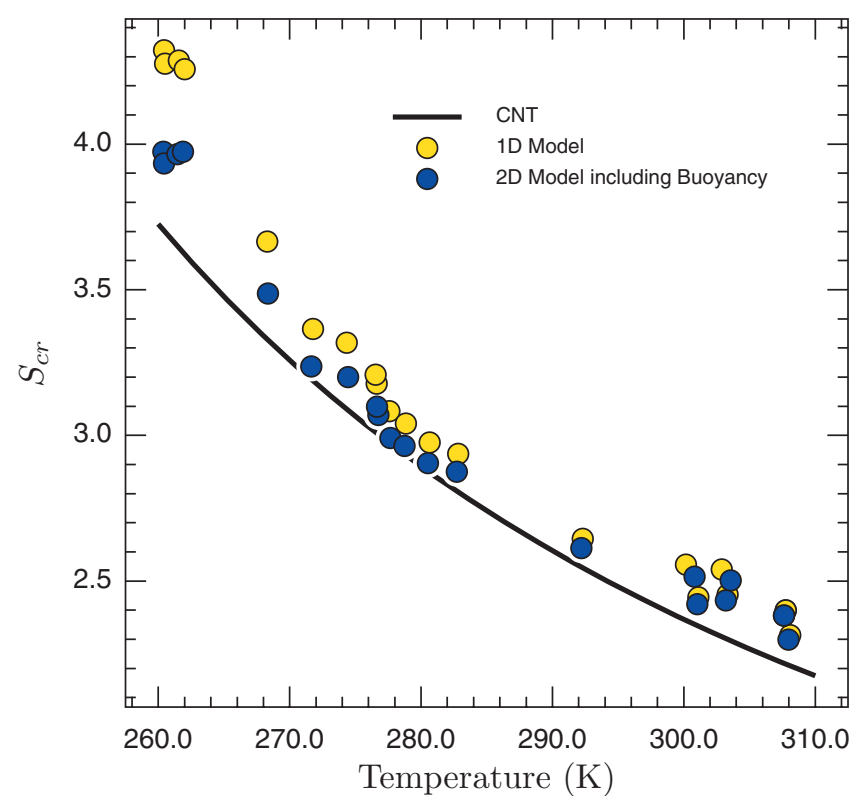

FIG. 3. n-propanol nucleation critical supersaturation data taken by Kacker and Heist and analyzed via the typical 1D model and the 2D model that includes buoyancy effects.

measurements as a function of supersaturation at three different isotherms. These rate measurements will be addressed later in this paper. As a first test of the influence of buoyant convection, the critical supersaturation data will be examined. These original critical supersaturation data are shown as the light yellow circles in Fig. 3. Previously, there seemed to be a discontinuity in the data near $275 \mathrm{~K}$. The data below $275 \mathrm{~K}$ seemed to follow a trend with a much steeper slope than the remaining, higher temperature data. Because of this discontinuity, Kacker and Heist fitted these two sections of the data to different curves. These data were reevaluated with the 2D model assuming ideal heating of the chamber sidewall and the results are given as the solid circles in Fig. 3. While the points at the very highest temperatures are essentially unaffected by buoyant convection there is a general lowering of the critical supersaturation with decreasing temperature. Moreover, the discontinuity at $275 \mathrm{~K}$ that had appeared with the 1D model has diminished and the data now appear to follow a smooth trend with the updated calculations. At the very lowest temperature, the shift in critical supersaturation is approximately $8 \%$. A table of the data presented in Fig. 3 is available in the supplementary information available online. ${ }^{48}$ At the time the data were taken, many of the criteria for stable TDCC operation had not been developed. Since that time, experimental observations have shown that chamber stability is favored by higher temperatures. ${ }^{10}$ One other point to note is that while the shift in supersaturations is significant, the variation in the computed temperatures between the 1D and 2D models does not appear to be very large. Often, with nucleation rate measurements in a TDCC, plate conditions are adjusted to maintain a constant nucleation temperature according to the typical 1D chamber model. At least in this particular case with propanol, it appears that convection has less of an influence on the temperature field and that these values are essentially the same as those calculated with 1D model. This is important to note 


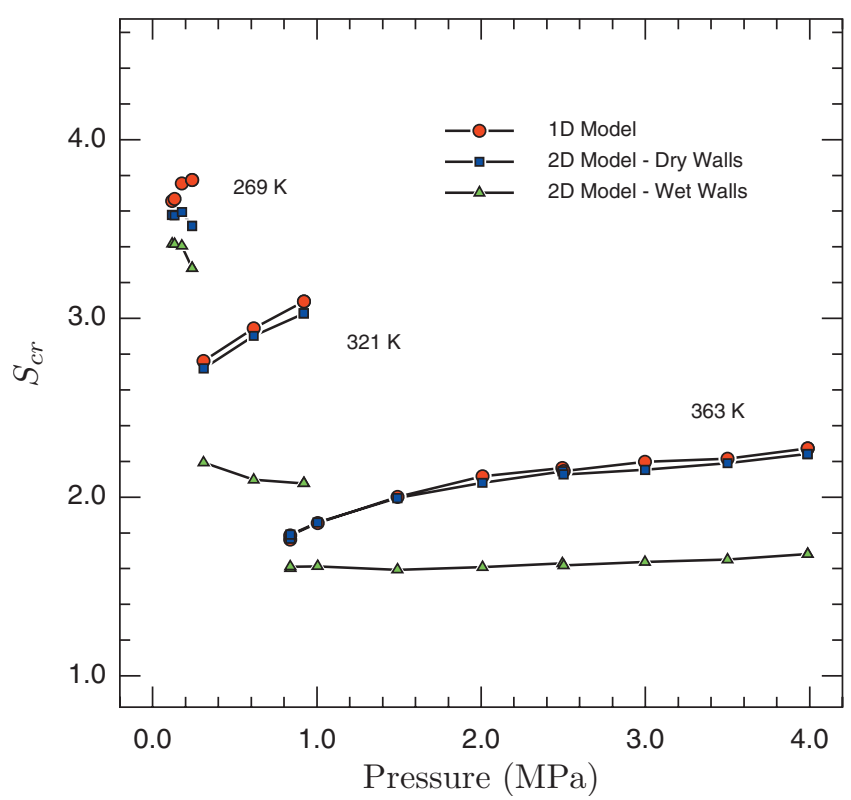

FIG. 4. Critical Supersaturation vs pressure for $n$-propanol in helium based on three different models of the chamber sidewall conditions.

because nucleation rate measurements taken at several isotherms will be examined later and these temperatures should remain unchanged and only the supersaturation values should be shifted.

\section{1994 HIGH PRESSURE CLOUD CHAMBER MEASUREMENTS}

In 1994, Heist et al. studied the nucleation of $n$-propanol over an extended pressure range using a special TDCC designed for high pressure, the high pressure cloud chamber. Propanol critical supersaturations up to $4 \mathrm{MPa}$ at constant temperatures were measured using both hydrogen and helium as carrier gases. In all cases, an increase in the calculated critical supersaturation was found with increasing pressure. The slope of this pressure dependence also increased with decreasing temperatures for both carrier gases. Finally, the slope of the pressure dependence at the same computed isotherm was different for the two carrier gases, with the stronger effect seen for helium.

The 2D model has been applied to three representative isotherms from Heist et al.'s original work with propanol in helium. In Heist et al.'s original work, the authors paid close attention to the effect of pressure upon the physical properties used in the calculation of the supersaturations because of the wide range of pressure investigated. In addition, over the extended range of pressure in this work, the vapor enhancement and surface tension effects noted by Luijten et al. may also play an important role. ${ }^{3,5}$ In this analysis, the focus is simply to examine the possible scope and changes in supersaturation values due to convection within the chamber. Therefore, physical properties for the propanol-helium case used in the 2D model are the same as used in Sec. V and given in Ref. 36.

The critical supersaturations calculated via a $1 \mathrm{D}$ analysis and a 2D analysis with wet and dry walls are shown in Fig. 4. As before, the dry operation is approximated assuming ideal heating of the chamber sidewall. In Sec. V, the greatest deviation between these ideal heating conditions and the typical 1D model occurs at the lowest temperature. This is also the case in Fig. 4. For the two highest isotherms, the ideal heating and 1D models agree very closely and both computed values increase with increasing pressure. At the $269 \mathrm{~K}$ isotherm, the available pressure range is much smaller, but there is a noticeable difference in the critical supersaturations from $1 \mathrm{D}$ and 2D dry wall models. These calculations show that under the assumption of an ideally heated, dry wall, there is, in general, still an apparent increase in critical supersaturation with pressure.

The third set of curves shown in Fig. 4 are the calculated critical supersaturations based on the assumption of wet walls. These values are significantly different from the other two calculations. Without knowing the conditions at the chamber wall, the ideal heating assumption is probably the best approximation to the sidewall conditions. In actual operation, the wall will be slightly overheated, resulting in perhaps slightly lower critical supersaturations. Therefore, at least with respect to convection, the ideal heating results can be viewed as an upper limit to the computed supersaturations. For the chamber conditions, no wall heating (i.e., wet wall operation) typically produces stronger convective currents due to the stronger density gradients at the sidewall. Since in the original experiment the chamber walls were heated, the supersaturation values are certainly likely to be larger than the values computed under this assumption. These two cases, again with respect to convection, represent upper and lower limits to the computed supersaturations, with the actual values likely being much closer to the ideal heating result.

For the wet wall case, the computed supersaturations are either essentially flat or, in fact, decreasing with pressure. It is important to note that all three cases are computed with the same code, geometry and physical properties, etc. The only difference in these cases is the approximation used for the chamber sidewall boundary conditions. These calculations show that it is certainly within the scope of buoyant convection to explain at least some of the observed pressure effect. Unfortunately, without more detailed information on the actual sidewall conditions, the effect of convection over these extended ranges of pressure cannot be accurately quantified to see if a pressure effect still remains.

\section{2006 PROPANOL TDCC MEASUREMENTS}

Brus et al. recently presented $n$-propanol nucleation rate measurements at various isotherms and chamber pressures. One of the objectives of this work was to measure the nucleation rate as a function of pressure to observe any pressure effect and investigate whether such variation could be explained with a more complete, 2D model of the chamber. These authors paid close attention to limit their experimental range to those conditions that met the strictest chamber stability criteria as specified in Refs. 10, 38, and 39. These stability criteria are based on ensuring that density inversions do not occur within the chamber, i.e., that the density of the vapor/carrier gas mixture is always decreasing with height. 


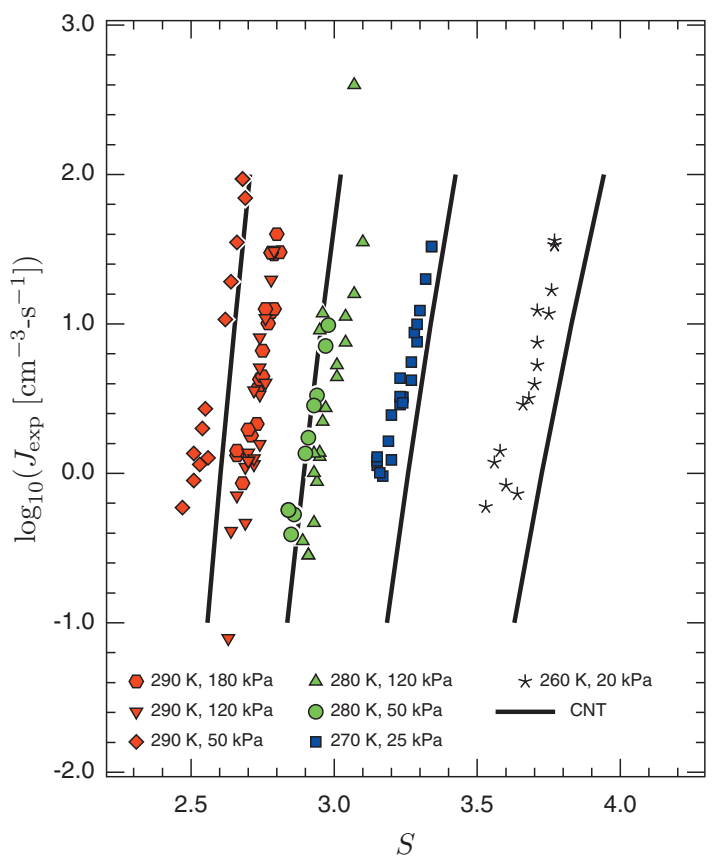

(a) Original Data

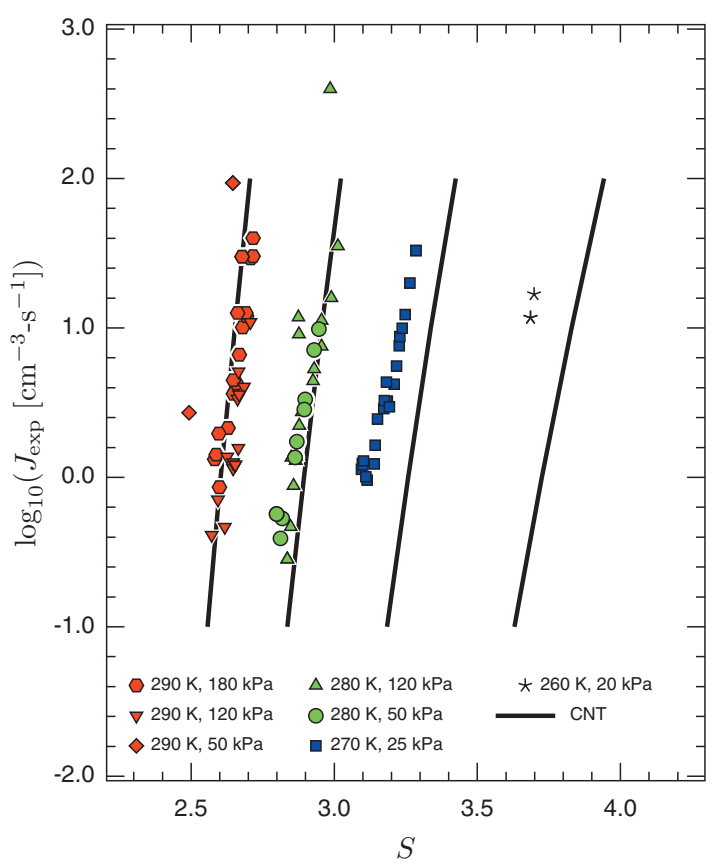

(b) Data recalculated using 2D model

FIG. 5. n-propanol in helium nucleation rate data taken by Brus et al. at four different isotherms and various pressures. The data shown in (a) are the data originally reported by Brus et al. The data shown in (b) are the results from the reanalysis of the original data using the $2 \mathrm{D}$ model described in this work.

Certainly, such an inversion would lead to convection within the chamber. It is important to note that even in cases where the density is at every point decreasing with height, that radial density gradients at the sidewall may still induce convective flows, although these flows will certainly be smaller in magnitude than those given by a density inversion. Natural or buoyant convective flows will work to stratify the density field within the entire chamber, including the sidewall where these radial density gradients exist.

In addition to the nucleation rate measurements, Brus et al. also performed various direct tests of the pressure effect. The focus of this reevaluation will be the propanol nucleation rate measurements that are shown in Fig. 5(a). Nucleation rate data were taken at four different isotherms$260,270,280$, and $290 \mathrm{~K}$. At the higher isotherms, the authors also took data at different pressures. Brus et al. noted a clear difference among the results due to pressure. As shown in Fig. 5(a), there is a slight difference between the 50 and 20 $\mathrm{kPa}$ data at $280 \mathrm{~K}$. Also, it should be noted that there is one high rate value at $280 \mathrm{~K}$, perhaps due to a typographical error in the table presented by Brus et al. At $290 \mathrm{~K}$, there is a distinct difference between the lowest pressure data and the remaining $290 \mathrm{~K}$ data. Brus et al. also noted that the observed differences in rate at different pressures could not be explained by reanalyzing the data with a more sophisticated, 2D model of the chamber.

As a preliminary test, the data presented by Brus et al. were first recalculated using the experimental conditions listed by Brus et al., but using the physical properties for the $n$-propanol-helium mixture from Kacker and Heist. ${ }^{36}$ This led to slightly different results than originally presented by Brus et al. so a comparison of the physical properties was made. All properties that are typically used in the $1 \mathrm{D}$ calcu- lation were the same except the binary diffusion coefficient and the thermal diffusion factor. The binary diffusivity used by Brus et al. appeared to be based on the Chapman-Enskog formula and was approximately $20 \%$ higher than the value used by Kacker and Heist. The thermal diffusion coefficient was given by a more complicated formula, but the changes noted between the data were likely beyond those attributable to this quantity.

A closer look showed that the reported propanol vapor viscosity was very large and looked to be in the form of perhaps a liquid viscosity. At first glance this may not seem to affect a 1D calculation of the chamber profiles since the momentum equations are not used in such an analysis. In the calculation of the temperature profile in a 1D calculation, the vapor-background gas mixture thermal conductivity is typically calculated via the Wassiljewa equation. ${ }^{40}$ In this equation, the mixture thermal conductivity depends not only on the mole fractions and individual, pure-component thermal conductivities, but also on the pure component vapor viscosities as well. Therefore, the value of this large viscosity typically would lead to an incorrect value of the mixture thermal conductivity. This error would likely be difficult to identify in a mixture thermal conductivity routine in a computer code because such a routine would report correct values at the extreme end points (pure vapor or pure background gas) and likely reasonable looking intermediate values.

Although no attempt was made to recalculate the data from Brus et al. using this large propanol viscosity to try to match the original results, it is noted that the nucleation data reported by Brus et al. do seem to support such a hypothesis. The greatest deviation in the data would likely occur when 
the propanol concentration in the chamber was high —at high temperatures and low pressures, the same conditions where the largest deviations are reported to occur.

Brus et al.'s original data have been recalculated using the physical properties for the $n$-propanol/helium system as given by Kacker and Heist. ${ }^{36}$ All of the values have been calculated using the $2 \mathrm{D}$ model of the chamber, using the chamber dimensions and experimental conditions reported by Brus et al., and assuming ideally heated chamber sidewalls. The results of these calculations are shown in Fig. 5(b). Previously, conditions in the chamber were tailored so that the experimental data fell at specific isotherms. With the recalculation of the data, these points are now scattered about these isotherms. A balance between setting very tight isotherms and plotting a reasonable portion of the data had to be made. It was found that plotting the data with $\pm 0.8 \mathrm{~K}$ spread about the original isotherm allowed at least two points from each of the original temperature and pressure data sets to be plotted. If data fell outside of this range, it was simply not plotted in Fig. 5(b).

The bulk of the data does, in fact, fall within this filtering window. In general, the values of supersaturation have shifted lower. While there was a slight difference in the values at different pressures at $280 \mathrm{~K}$, these two data sets are essentially identical with the updated calculations. Only two data points from the $50 \mathrm{kPa}, 290 \mathrm{~K}$ data set remain because most of these values actually correspond to approximately $291 \mathrm{~K}$ and higher with the updated calculations. Even so, the higher pressure $290 \mathrm{~K}$ data have also shifted closer to these lower pressure values. At the other end of the experimental temperature range, only two data points at the $260 \mathrm{~K}$ isotherm fall within the filtering window; most of these data points correspond to a temperature of $259 \mathrm{~K}$ and lower. Interestingly, the data at 280 and $290 \mathrm{~K}$ now seem to agree extremely well with the CNT predictions. Another point to notice is that even though the allowable temperature window for plotting the data has increased (i.e., values within $\pm 0.8 \mathrm{~K}$ of the isotherm are now plotted), the scatter in the data has not increased. In fact, using the $270 \mathrm{~K}$ isotherm as an example, the scatter in the values seems to have even decreased slightly.

The shifts in the data from Figs. 5(a) and 5(b) are due to two factors. The predominant factor in the temperature shift is due to the incorrect transport property. For example, there is a shift of as much as $2.6 \mathrm{~K}$ in the calculated nucleation temperature and a shift of 0.07 in calculated supersaturation between the values reported by Brus et al. and those calculated with a 1D model and physical properties from Ref. 36. A second shift in the data is due to buoyant convection. This convection has a much weaker effect on the calculated temperatures, but a comparable effect on the supersaturation. For example, the difference between the corrected 1D model and the 2D model yields only a maximum temperature difference of $0.3 \mathrm{~K}$ in calculated temperatures, but a difference of 0.09 in calculated supersaturation. When these two factors are considered together, the maximal differences between the current 2D model and the data reported by Brus et al. amount to $2.5 \mathrm{~K}$ in calculated temperatures and 0.12 in supersaturation (approximately 5\%). A complete list of the data

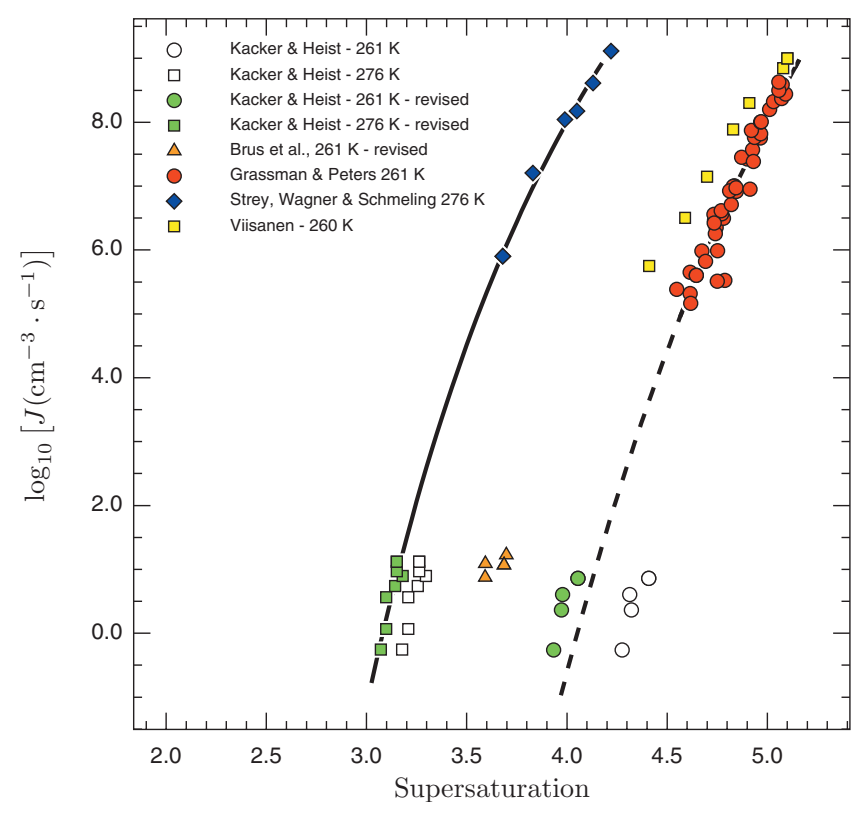

FIG. 6. Comparison of nucleation flux data for overlapping isotherms for TDCC studies and expansion-based studies.

calculated via the 1D model, a 2D model excluding buoyancy, and the 2D model including buoyancy is given as a supplementary table available online. ${ }^{48}$

\section{COMPARISON WITH EXPANSION-BASED STUDIES}

Certainly, one goal in experimental nucleation research is to provide data over a wide range that can be used as a test for nucleation theories. For propanol, there is only a small overlap in the available experimental temperature range for cloud chamber investigations and expansion-based devices. In addition to this small temperature range, another problem in making such comparisons is finding nucleation rate data at identical isotherms.

One such overlapping isotherm is the $276 \mathrm{~K}$ data from Strey et $a ._{.}{ }^{41}$ and Kacker and Heist. ${ }^{36}$ The data from Strey et al. were taken using a two piston expansion chamber with argon as the background gas and these data are shown in Fig. 6. Kacker and Heist took data for propanol at three different isotherms with helium as the background gas. These data were originally taken up to high nucleation rate levels where latent heat and vapor depletion effects become significant. The data at these high rates $\left(>100 \mathrm{~cm}^{-3} \mathrm{~s}^{-1}\right)$ were analyzed using a model that corrected for these effects. In this work, only the lower rate values where these vapor depletion and latent heat effects are negligible are considered. The data from Kacker and Heist at $276 \mathrm{~K}$ analyzed using the 1D model of the chamber are shown as the open squares in Fig. 6. Based on the reanalysis of the critical supersaturation data given earlier, it is expected that the recalculated data should remain at the $276 \mathrm{~K}$ isotherm, but the values are shifted to lower values of the supersaturation. These new values are shown as the green squares in Fig. 6. As before, these values were calculated using the $2 \mathrm{D}$ model with the assumption of ideal heating at the chamber sidewalls. The agreement be- 
tween the two different sets appears to be very good and it is interesting to note that these two data sets were taken with different background gases.

One method for fitting experimental nucleation rate measurements is to fit the experimental data to the following equation: ${ }^{42}$

$$
J=K S^{2} \exp \left[\frac{-E^{3}}{2 \ln ^{2} S}\right] .
$$

In this equation, $J$ is the nucleation rate and $S$ is the supersaturation. The parameter $K$ represents the temperaturedependent, pre-exponential factor from CNT except for the supersaturation $S^{2}$ term, while the parameter $E$ is the exponential term of CNT, again excluding the supersaturation term. This parameter $E$ is the temperature-dependent surface energy of a cluster of radius $r$ and surface tension $\sigma$ to $3 / 2 k T,{ }^{42}$

$$
E=\frac{4 \pi r^{2} \sigma}{3 / 2 k T}
$$

An unweighted fit to the $276 \mathrm{~K}$ data of Strey et al. and Kacker and Heist gives the solid line shown in Fig. 6 with parameters $K=7.14 \times 10^{21} \mathrm{~cm}^{-3} \mathrm{~s}^{-1}$ and $E=5.107$. Again, both sets of data follow this curve very closely and it should be reiterated that the two data sets were taken with different background gases.

There is one additional overlapping isotherm, but the picture is less clear in this case. Graßmann and Peters ${ }^{42}$ collected numerous data points at $261 \mathrm{~K}$, shown in Fig. 6 as the red circles. As in the case of the $276 \mathrm{~K}$ data set, the $261 \mathrm{~K}$ data points from Kacker and Heist have been reanalyzed and are shown as the open and green circles for the 1D and 2D models, respectively.

There is a larger shift in the Kacker and Heist supersaturation values for the $261 \mathrm{~K}$ data than for the $276 \mathrm{~K}$ data. This larger shift would have been expected from the critical supersaturation results shown in Fig. 3. Graßmann and Peters presented a fit to Eq. (14) for their $261 \mathrm{~K}$ data and this fit has been extrapolated down to the lower rate values available with the TDCC, shown in Fig. 6 as the dashed line. The TDCC $261 \mathrm{~K}$ data are just slightly below this extrapolation, but overall there appears to be reasonable agreement between these two data sets. A new fit to both of these studies alone would likely only be slightly different than the values reported by Graßmann and Peters. It should be noted that the results from Graßmann and Peters were also taken with a different background gas (nitrogen) than either used by Strey et al. (argon) or Kacker and Heist (helium).

Unfortunately, the overall agreement between the data at the lower temperature is not as good as the $276 \mathrm{~K}$ values. Brus et al. ${ }^{15}$ took data at $260 \mathrm{~K}$, but after the reanalysis of the data given earlier, several points now fall at the $261 \mathrm{~K}$ isotherm and are plotted as the yellow triangles in Fig. 6. These points are lower than the values of Kacker and Heist by approximately 0.3 supersaturation units. In addition to these differences in TDCC studies at this isotherm, there is also a difference at the higher rate, expansion-based data in this temperature range. Viisanen ${ }^{43}$ took data for $n$-propanol at $260 \mathrm{~K}$, very close to the $261 \mathrm{~K}$ data of Graßmann and Peters, but these supersaturation values are less than the values reported by Graßmann and Peters. Therefore, there is a larger fluctuation in the $n$-propanol nucleation data near the $261 \mathrm{~K}$ isotherm at both the nucleation rate ranges available via the TDCC and expansion-based devices.

\section{COMPARISON WITH HALE THEORY}

A direct comparison between the TDCC and expansion studies is difficult because of both the narrow overlapping temperature range and the available isotherms. In addition there are several other sources of $n$-propanol nucleation data available in the literature that fall outside of this temperature window. One convenient method of comparing all of these experimental data is via Hale's scaled nucleation theory (SNT). ${ }^{44}$ This theory uses scaled and critical point quantities to recast $\mathrm{CNT}$ in a form where a wide range of experimental conditions can be compared in a single plot.

Hale's SNT has the following form:

$$
J_{\text {scaled }}=J_{0 c} \exp \left(\frac{16 \pi}{3} \Omega^{3}\left[\frac{T_{c}}{T}-1\right]^{3} \frac{1}{(\ln S)^{2}}\right),
$$

where $J_{\text {scaled }}$ is the nucleation rate, $J_{0 c}$ is a kinetic prefactor given by the nucleation rate evaluated at the critical temperature $T_{c}$, and assigned the value of $10^{26} \mathrm{~cm}^{-3} \mathrm{~s}^{-1}$. The quantity $\Omega$ termed the excess surface entropy per molecule (divided by k) is a parameter that ranges from 1.5 to 2.2. If experimental nucleation data are plotted in the form $-\log \left(J / 10^{26}\right)$ versus $C_{0}\left[\left(T_{c} / T\right)-1\right]^{3} /(\ln S)^{2}$, and the value of $C_{0}$ adjusted to minimize the error between a line of slope $=1$ passing through the origin, then the value of $\Omega$ can be found from

$$
\Omega=\left(\frac{3 C_{0}}{16 \pi} \cdot \ln 10\right)^{1 / 3} .
$$

The advantage of the term $C_{0}\left[\left(T_{c} / T\right)-1\right]^{3} /(\ln S)^{2}$ is that it accounts for both the temperature and supersaturation dependence in the exponential of the nucleation rate expression, allowing a wide range of nucleation conditions to be shown on a single plot. Such a plot has been constructed for $n$-propanol by several researchers in the past and only slight differences due to the recalculated TDCC results are expected. Nevertheless, for completeness the updated plot is given in Fig. 7. In this figure, all the nucleation data for $n$-propanol that is available from the literature have been plotted. These data include the expansion-based studies of Graßman and Peters, ${ }^{42}$ Strey et al., ${ }^{41}$ Viisanen, ${ }^{43}$ Viisanen et al., ${ }^{45}$ TDCC data taken by Kacker and Heist ${ }^{36}$ and Brus et al., ${ }^{15}$ and supersonic nozzle data taken by Gharibeh et al. ${ }^{46}$ and Ghosh et al. ${ }^{47}$

A couple of notes should be made regarding the data used in Fig. 7. First, only those data following the restriction imposed by Hale's SNT, namely, $\left(T_{c} / T<0.5\right)$, are given in the plot. For the TDCC studies, this means that only the lowest available temperatures are included. In addition, for the TDCC studies only the nucleation rate measurements as a function of supersaturation are plotted. Therefore, the data from Figs. 6 and 5(b) are included, but the critical supersatu- 


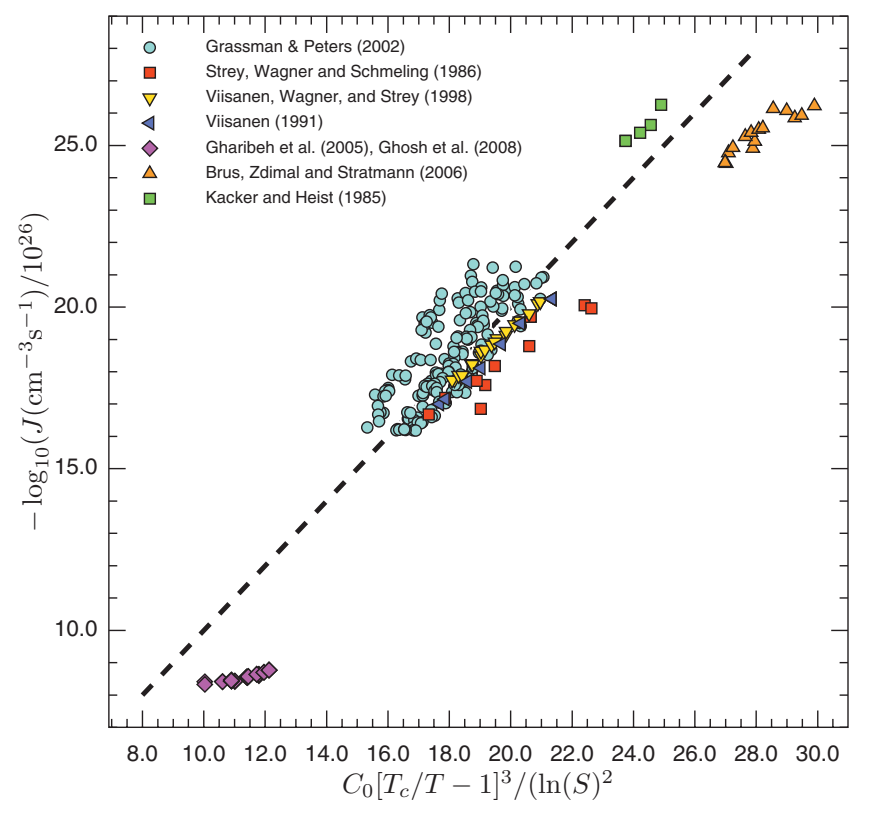

FIG. 7. SNT plot for $n$-propanol nucleation data.

ration data from Figs. 3 and 4 are not. Earlier, only two points were included in Fig. 5(b) of the recalculated data from Brus et al. because the nucleation temperatures fell outside the nominal $260 \mathrm{~K}$ isotherm. These additional points can now be included in Fig. 7. Because of the number of data points, Graßmann and Peters only provided fits to their propanol data, but in Fig. 7 their original data points have been used in the plot as well as in the construction of the fit. Finally, the computed supersaturation values are very dependent on the vapor pressure expression used. Therefore, each work shown in the graph was checked to verify that the same vapor pressure expression was used.

The value of $C_{0}$ was calculated by performing an unweighted fit to all of the data shown in Fig. 7. This value was found to be 39.0, giving a value of $\Omega$ of 1.75 . As expected, these values are only slightly different from the values of 37.5 and 1.73 reported from the most recent fit. ${ }^{46}$ In this previous Hale plot, most of the TDCC results fell at or above the line given by Hale theory where in this case the TDCC results now straddle the fit. In addition, the data from both TDCC results seem to have slopes consistent the SNT fit.

\section{CONCLUSIONS}

Previous studies of buoyant convection in a TDCC have focused on elucidating the role convection can play with these devices and how to minimize these flows. In this work the goal was to apply such a model to specific TDCC studies, recalculating the results to account for such flows. The model was applied to three different TDCC studies of $n$-propanol. In general, after correcting for buoyant effects there were improvements in the results. In the first TDCC set of experiments the model appeared to resolve a discontinuity in critical supersaturation data. In this same study, the effect of buoyancy on isothermal nucleation rate measurements was also studied. In this case, the computed supersaturations were shifted to lower values, putting these data in reasonable agreement with expansion data taken at the same temperature.

In the second TDCC study, the model was applied to nucleation data taken for $n$-propanol over an extended pressure range. The focus of this study was to examine whether buoyant convection could possibly explain the observed pressure dependence. Because the sidewall boundary conditions are typically not measured, these values cannot be calculated with certainty. Nevertheless, using simple models of wall conditions that should offer limits to the range of values, it was found that convection could possibly account for some of the observed pressure effect.

In the third TDCC study, a pressure effect was originally noted for the higher temperature, lower pressure ranges of the data. This perceived pressure effect disappeared after correcting for buoyancy and an incorrect transport property.

A comparison of propanol nucleation data taken at the same temperatures, but in different experimental devices. After correcting the TDCC data for buoyancy effects, there was some improvement in the agreement between the two types of experimental devices, especially at the highest available temperature. At the lower temperature, some inconsistencies still exist between the two types of devices.

A direct comparison of nucleation data between different experimental devices is often difficult because the window of overlapping experimental conditions is often small. SNT allows a much wider amount of data to be compared in a single plot. Such a plot was constructed with the TDCC data corrected for buoyancy and other $n$-propanol nucleation data available in the literature. In general, the agreement between data and SNT is good. In the most recent comparison between $n$-propanol nucleation data and scaled nucleation, essentially all of the TDCC data fell at or above the predictions of SNT. After correcting for buoyancy, these TDCC data now straddle the predictions of SNT, with each set of data having a similar slope to the SNT prediction.

One goal of this work was to demonstrate via quantitative calculations the role buoyancy can have on TDCC results and offer further evidence that buoyancy may be a possible explanation for the observed pressure effect in these devices. Currently, there is no way to determine with certainty if buoyancy is responsible for the pressure effect in TDCCs because these flows are generated entirely by conditions at the chamber sidewall that are currently never measured. Most of the computations within this work have been performed under the assumption of ideal heating at the chamber wall. While this is likely a reasonable assumption, there may be cases where the chamber wall is overheated and this situation would likely result in even lower actual supersaturations. It would be interesting to perform some TDCC experiments while monitoring and correcting for wall conditions to see if a pressure effect remains.

\section{ACKNOWLEDGMENTS}

We wish to thank Dr. Franz Peters for supplying us with his $n$-propanol nucleation data. 
${ }^{1}$ R. H. Heist, M. Janjua, and J. Ahmed, J. Phys. Chem. 98, 4443 (1994).

${ }^{2}$ R. H. Heist, J. Ahmed, and M. Janjua, J. Phys. Chem. 99, 375 (1995).

${ }^{3}$ C. C. M. Luijten, P. Peeters, and M. E. H. van Dongen, J. Chem. Phys. 111, 8535 (1999).

${ }^{4}$ P. Peeters, J. Hrubý, and M. E. H. van Dongen, J. Phys. Chem. 105, 11763 (2001).

${ }^{5}$ C. C. M. Luijten and M. E. H. van Dongen, J. Chem. Phys. 111, 8524 (1999).

${ }^{6}$ J. A. Fisk and J. L. Katz, J. Chem. Phys. 104, 8649 (1996).

${ }^{7}$ I. J. Ford, in Nucleation and Atmospheric Aerosols, edited by N. Fukuta and P. E. Wagner (Deepak, Hampton, VA, 1992)

${ }^{8}$ D. W. Oxtoby and A. Laaksonen, J. Chem. Phys. 102, 6846 (1995).

${ }^{9}$ D. Kane, S.P. Fisenko, M. Rusyniak, and M.S. El-Shall, J. Chem. Phys. 111, 8496 (1999).

${ }^{10}$ A. Bertelsmann and R. H. Heist, J. Chem. Phys. 106, 624 (1997).

${ }^{11}$ F. T. Ferguson, R. H. Heist, and J. A. Nuth, J. Chem. Phys. 115, 10829 (2001)

${ }^{12}$ F. Stratmann, M. Wilck, V. Ždímal, and J. Smolík, J. Phys. Chem. B $\mathbf{1 0 5}$, 11641 (2001).

${ }^{13}$ N. Schaeffer, F. Utheza, F. Garnier, and G. Lauriat, J. Chem. Phys. 113, 8085 (2000)

${ }^{14}$ A. L. Itkin, Aerosol Sci. Technol. 34, 479 (2001).

${ }^{15}$ D. Brus, V. Ždímal, and F. Stratmann, J. Chem. Phys. 124, 164306 (2006).

${ }^{16}$ J. Merikanto, E. Zapadinsky, and H. Vehkammäki, J. Chem. Phys. 125, 084503 (2006).

${ }^{17}$ H. Y. Tang and I. A. Ford, J. Chem. Phys. 125, 144316 (2006).

${ }^{18}$ K. Yasuoka and X. C. Zeng, J. Chem. Phys. 126, 124320 (2007).

${ }^{19}$ J. Wedekind, D. Reguera, and R. Strey, J. Chem. Phys. 127, 064501 (2007).

${ }^{20}$ J. C. Barrett, J. Chem. Phys. 128, 164519 (2008).

${ }^{21}$ J. Wedekind, A.-P. Hyvärinen, D. Brus, and D. Reguera, Phys. Rev. Lett. 101, 125703 (2008).

${ }^{22}$ D. Mitrakos, V. Ždímal, D. Brus, and C. Housiadas, J. Chem. Phys. 129, 054503 (2008).

${ }^{23}$ A.-P. Hyvärinen, D. Brus, V. Ždímal, J. Smolík, M. Kulmala, Y. Viisanen, and H. Lihavainen, J. Chem. Phys. 124, 224304 (2006).

${ }^{24}$ A.-P. Hyvärinen, D. Brus, V. Ždímal, J. Smolík, M. Kulmala, Y. Viisanen, and H. Lihavainen, J. Chem. Phys. 128, 109901 (2008).

${ }^{25}$ E. Herrmann, H. Lihavainen, A.-P. Hyvärinen, I. Riipinen, M. Wilck, F. Stratmann, and M. Kulmala, J. Phys. Chem. A 110, 12448 (2006).

${ }^{26}$ E. Herrmann, A.-P. Hyvärinen, D. Brus, H. Lihavainen, and M. Kulmala,
J. Phys. Chem. A 113, 1434 (2009).

${ }^{27}$ D. Brus, A.-P. Hyvärinen, J. Wedekind, Y. Viisanen, M. Kulmala, V. Ždímal, J. Smolík, and H. Lihavainen, J. Chem. Phys. 128, 134312 (2008).

${ }^{28}$ F. T. Ferguson and J. A. Nuth, J. Chem. Phys. 111, 8013 (1999).

${ }^{29}$ J. A. Fisk, V. M. Chakarov, and J. L. Katz, J. Chem. Phys. 104, 8657 (1996).

${ }^{30}$ M. P. Anisimov, E. G. Fominykh, S. V. Akimov, and P. K. Hopke, J. Aersol. Sci. 40, 733 (2009).

${ }^{31}$ M. P. Anisimov, S. D. Shandakov, Yu. I. Polygalov, and R. H. Heist, J. Chem. Phys. 114, 899 (2001).

${ }^{32}$ F. T. Ferguson, R. H. Heist, and J. A. Nuth, J. Chem. Phys. 113, 7398 (2000).

${ }^{33}$ S. Patankar, Numerical Heat Transfer and Fluid Flow (Hemisphere, Washington, DC, 1980).

${ }^{34}$ D. J. Tritton, Physical Fluid Dynamics (Clarendon, New York, 1988).

${ }^{35}$ M. M. Rudek, J. L. Katz, I. V. Vidensky, V. Ždímal, and J. Smolík, J. Chem. Phys. 111, 3623 (1999).

${ }^{36}$ A. Kacker and R. H. Heist, J. Chem. Phys. 82, 2734 (1985).

${ }^{37}$ R. B. Bird, W. E. Stewart, and E. N. Lightfoot, Transport Phenomena (Wiley, New York, 1960)

${ }^{38}$ J. L. Katz, J. Chem. Phys. 52, 4733 (1970).

${ }^{39}$ A. Bertelsmann and R. H. Heist, J. Chem. Phys. 106, 610 (1997).

${ }^{40}$ R. C. Reid, J. M. Prausnitz, and B. E. Poling, The Properties of Gases and Liquids (McGraw-Hill, New York, 1987).

${ }^{41}$ R. Strey, P. E. Wagner, and T. Schmeling, J. Chem. Phys. 84, 2325 (1986).

${ }^{42}$ A. Graßmann and F. Peters, J. Chem. Phys. 116, 7617 (2002).

${ }^{43}$ Y. Viisanen, Ph.D. thesis, University of Helsinki, 1991; Commentationes Physico-Mathematicae et Chemico-Medicae 133, 37 (1991).

${ }^{44}$ B. N. Hale, Phys. Rev. A 33, 4156 (1986).

${ }^{45}$ Y. Viisanen, P. E. Wagner, and R. Strey, J. Chem. Phys. 108, 4257 (1998).

${ }^{46}$ M. Gharibeh, Y. Kim, U. Dieregsweiler, B. E. Wyslouzil, D. Ghosh, and R. Strey, J. Chem. Phys. 122, 094512 (2005).

${ }^{47}$ D. Ghosh, A. Manka, R. Strey, S. Seifert, R. Winans, and B. E. Wyslouzil, J. Chem. Phys. 129, 124302 (2008).

${ }^{48}$ See supplementary material at http://dx.doi.org/10.1063/1.3429618 for a comparison of temperature and supersaturation values computed from different models of the chamber. These data are used to construct Figs. 3 and 5 in the manuscript. 